\title{
Potential therapeutic effects of Nigella sativa and its constituents, thymoquinone on COVID-19 based on immunomodulatory effects
}

\author{
Mohammad Reza Khazdair ${ }^{1}$ \\ ${ }^{1}$ Birjand University of Medical Sciences
}

May 6, 2020

\begin{abstract}
The novel coronavirus (COVID-19) is a kind of viral pneumonia and emerging pathogen that causes a severe respiratory infection in humans. The research on COVID-19 is still in the primary stage and international attention has raised to develop treatment option. The main death cause of COVID-19 is acute respiratory distress syndrome (ARDS). The main mechanisms for ARDS is the cytokine storm that release the large amounts of pro-inflammatory cytokines. The other corona virus such as, severe acute respiratory syndrome coronavirus 2 (SARS-CoV-2) cause to reduction of CD4+ and CD8+ T cells in the peripheral blood of infected patients. Some medicinal plants produce essential oils that are used in traditional and modern medicine, and in the food, cosmetics, and pharmaceutical industry. We aimed to summarize the possible therapeutic effects of Nigella sativa and its constituents, thymoquinone based on immunomodulatory effects on COVID-19. The databases including; Web of Science (ISI), PubMed, Scopus and google scholar were used to search for keywords such as, COVID-19, Nigella sativa, thymoquinone, Antivirus, and Immunomodulatory effects. N. sativa extract and thymoquinone (TQ) reduced the level of cytokines including; IL-2, IL-4, IL-6, and IL-12, while enhanced IFN- $\gamma$ in the bronchoalveolar lavage fluid and serum. They also increased the serum levels of IgG1 and IgG2a. N. sativa and TQ decreased T helper 2 (Th2)/Th1 and Th2/ Regulatory T cells (Tregs) ratios due to elevation of IL-10 but suppression of IL-4. Then this medicinal plant and its constituents might be beneficial effects in control and treatment of COVID-19.
\end{abstract}

\section{Hosted file}

NS-Immune-COVID-19-BJCP.doc available at https://authorea.com/users/318471/articles/448349potential-therapeutic-effects-of-nigella-sativa-and-its-constituents-thymoquinone-oncovid-19-based-on-immunomodulatory-effects 